\title{
On LBNE Neutrino Flux Systematic Uncertainties
}

\author{
Paul L.G. Lebrun*, James Hylen*, Alberto Marchionni*, Laura Fields ${ }^{\dagger}$, Amit \\ Bashyal $^{* *}$, Seongtae Park ${ }^{* *}$ and Blake Watson** \\ *Fermi National Accelerator Laboratory, Batavia, Illinois 60510, USA \\ ${ }^{\dagger}$ Northwestern University, Evanston, Illinois 60208, USA \\ ${ }^{* *}$ Univ. of Texas (Arlington), Arlington, TX 76019, USA
}

\begin{abstract}
.
The systematic uncertainties in the neutrino flux of the Long-Baseline Neutrino Experiment, due to alignment uncertanties and tolerances of the neutrino beamline components, are estimated. In particular residual systematics are evaluated in the determination of the neutrino flux at the far detector, assuming that the experiment will be equipped with a near detector with the same target material of the far detector, thereby canceling most of the uncertainties from hadroproduction and neutrino cross sections. This calculation is based on a detailed Geant4-based model of the neutrino beam line that includes the target, two focusing horns, the decay pipe and ancillary items, such as shielding.
\end{abstract}

Keywords: Neutrino Beam Simulation

PACS: 10.13

\section{INTRODUCTION}

One of the most important goal of Long-baseline Neutrino Experiment (LBNE) [1] is the determination of the $\mathrm{CP}$ violating phase in the neutrino flavor mixing matrix, via the well-known neutrino oscillation phenomena. This measurement requires an accurate prediction of the neutrino and antineutrino fluxes, for both electron and muon species, at the far Detector (FD), starting from measurements in the near Detector (ND). At the first order, uncertainties in the flux will cancel in the ratio FD/ND, if the two detectors are made of the same material. But the neutrino beam profile at ND will be a bit different than at FD, as the latter detector sees basically a point source of neutrinos, while the former sees a more complicated beam geometrical structure. Here we will discuss the subtle effects in the difference of the neutrino flux between ND and FD induced by alignment uncertanties and tolerances of the neutrino beamline components, which can be estimated via Monte Carlo methods. The aim of this paper is to described the method and show results for the $700 \mathrm{~kW}$ target/horn system [2], proposed in the early phase of the experiment. A typical neutrino flux seen at the near detector is shown in figure 1

Moreover, being able to determine, ab initio, the flux of neutrino at the ND will allow us to study neutrino interactions with nuclei, thereby enhancing our understanding of the nuclear structure in a unique and precise way. As the knowledge of hadro-production of pions and kaons improves, it does make sense to improve the technology and accuracy of the neutrino beam simulations, ultimately opening the field of high precision electro-weak nuclear physics at the $\approx \mathrm{GeV}$ scale.

\section{METHOD}

The Geant 4 toolkit [3] has been extensively used to simulate neutrino beams, including the existing Neutrino at the Fermilab Main Injector (NUMI) beam line [4]. This work started prior to the High Energy Physics "Snowmass 2013" meeting ${ }^{1}$. At that time, the LBNE collaboration supported the idea to start their experimental program with the socalled $700 \mathrm{~kW}$ reference design of the beam line [2]. This design uses the well tested NuMI beam line components, such as the $700 \mathrm{~kW}$ target and the two-horn focusing system. While the results presented here are limited to this configuration, the methods and software packages will be used for future optimized neutrino beam line designs.

1 see http://www.snowmass2013.org/tiki-index.php 


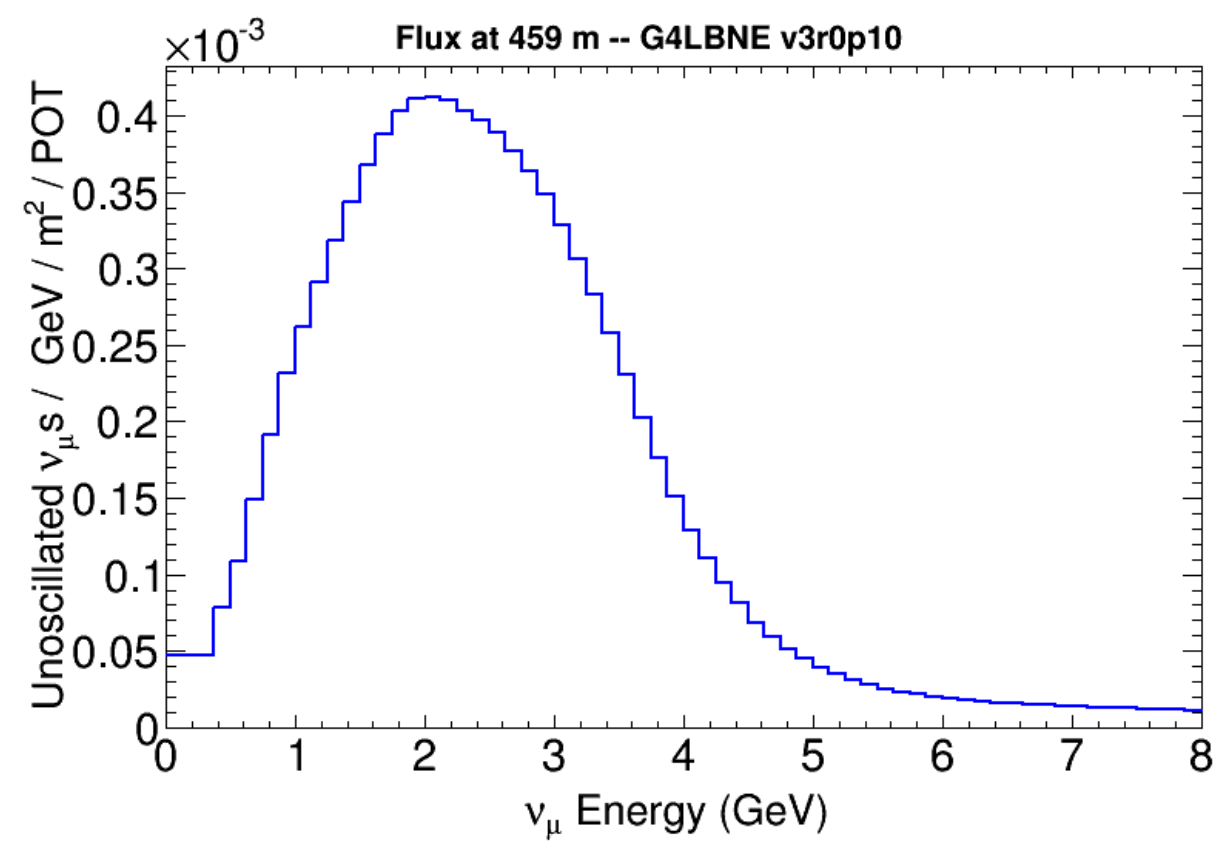

FIGURE 1. Typical Neutrino flux observed at a generic ND

A Geant4 implementation, called G4NuMI, of this beam line existed, along with a set of analysis tools allowing us to determine the flux at the ND or FD. However, in G4NuMI, and its subsequent incarnation dubbed G4LBNE, the validation of a small changes in the geometry of the beam line elements, such as those due to misalignments, for instance, became a difficult task, mostly due to the usage of outdated configuration files, and/or conflict the use of the Geant4 User Interface (G4UI) commands. The following upgrades to G4LBNE were implemented:

- Removal of the old and obsolete (as its design and usage dated from the Geant3 days) NuMI data card file. All setting changes are now introduced via the formal G4UI. While default values for all the parameters of the simulation are hard-coded, those which are relevant and/or adjustable by construction (such as the longitudinal position of the target) are uploaded in the G4UI classes and therefore available to the user while running G4LBNE interactively.

- Creation of a "LBNE-Surveyor" class, along with ad hoc G4UI commands, allowing the users to simulate misalignments.

- Creation of an "LBNEVolumePlacement" class, which interfaces between the LBNESurveyor class and the uploading of the geometry as the usual Geant4 hierarchy of physical volumes.

- Expansion of the Geant4 "SteppingAction" class to allow for extensive debugging of the geometry, via trajectory ASCII dumps of muon or geantino tracks.

The implementation of the geometry (baffle, target, Horn1, Horn2, etc..) had therefore to coded again. It has been fully based on engineering drawings.

The G4LBNE program produces a listed of weighted neutrinos, along with information on the neutrino flavor and its ancestry, allowing us to determine the origin and decay point of the neutrino parent. The kinetic energy of the neutrino in the rest frame of its parent is also saved, allowing the calculation of the weight and energy for a possible detection of this neutrino at an arbitrary distance from the target and for a given solid angle, set by the detector size.

We relied on various techniques to validate G4LBNE:

- Code inspection by collaborators who are not the primary authors of the specific code under review.

- Standard benchmark: any significant change to the code is always followed by a comparison of the neutrino flux with the "nominal" beam line configuration flux. 
TABLE 1. Tolerance values for key parameters in the neutrino beam line

\begin{tabular}{lcc}
\hline \multicolumn{1}{c}{ Parameter } & Tolerance & units \\
\hline Target position, transv. (each end) & 0.5 & $\mathrm{~mm}$ \\
Target density, relative & 2 & $\%$ \\
Horn 1 position, transv. (each end) & 0.5 & $\mathrm{~mm}$ \\
Horn 2 position, transv. (each end) & 0.5 & $\mathrm{~mm}$ \\
Far detector position, transv. & 21 & $\mathrm{~m}$ \\
Near detector position, transv. & 25.5 & $\mathrm{~cm}$ \\
Decay pipe position, transv. & 2 & $\mathrm{~cm}$ \\
Decay pipe radius & 10 & $\mathrm{~cm}$ \\
Horn conductor skin depth & 6 & $\mathrm{~mm}$ \\
Horn current & 2 & $\mathrm{kA}$ \\
Horn water layer thickness & 0.5 & $\mathrm{~mm}$ \\
Beam spot size at target & 0.1 & $\mathrm{~mm}$ \\
Beam position at target & 0.45 & $\mathrm{~mm}$ \\
Beam angle at target & 70 & $\mu \mathrm{rad}$ \\
Baffle scraping & 0.25 & $\%$ \\
Misalignment of shielding blocks & 1 & $\mathrm{~cm}$ \\
\hline
\end{tabular}

* Assumed nominal value, it was varied up to infinity

$\dagger$ Out of $200 \mathrm{kA}$, or $1 \%$

- For any change in the geometry, two distinct approaches were used to verify that the volumes are of the specified size and position: interactive visualization and use of trace dumps (sets of $3 \mathrm{D}$ positions and named volume boundaries) for geantino particles.

\begin{abstract}
ASSUMPTIONS
The most relevant set of tolerances (allowed deviation from nominal) on critical parameters of the beamline components is given on table 1. These tolerance figures are based on previous study performed for the NuMI beamline [6].

For a given neutrino energy, we are primarily interested in the fractional change in the ratio of the weighted flux, ND/FD, due to a change in the parameter commensurate with the tolerance values listed on table 1 . Such calculations are done over a neutrino energy range of $10 \mathrm{GeV}$ with an energy bin size of $0.5 \mathrm{GeV}$. Thus, the double ratio, ND/FD, variant to Nominal is computed for 20 energy bins and, typically, 4 values of the deviations from nominal, for each parameters. Each parameters is treated separately. Correlation between observed deviations in the weighted neutrino fluxes due to correlated and simultaneous changes in the parameters were found to have a small impact on our final results.
\end{abstract}

\title{
RESULTS
}

An example of such a double ratio is shown on figure 2, varying this time the horizontal position of the target. The pion flux emerging from the target does change as the $7.4 \mathrm{~mm}$ wide target no longer intercepts the $\approx 1.3 \mathrm{~mm}$ (one $\sigma$ ) wide beam, if displaced with respect to this beam. Given the fact that the hadrons produced in the most upstream part of the target can re-interact in the downstream part, or the horns, this is not equivalent to a trivial calculation of the overlapping surface a 2D Gaussian with the rectanguler target shape. From this simulated data, via a simple polynomial fits, an upper limit on the fractional error on the weighted flux ratio ND/FD of $\approx 0.5 \%$ can be extracted, should the alignment of the target be better then $0.5 \mathrm{~mm}$. Again, this is done for each $0.5 \mathrm{GeV}$ bin, for the un-oscillated muon neutrinos.

Similarly, shown on figure 3, the effect of a horizontal misalignment of the first horn, either offset or tilt, has been studied in detail.

The relative change due to a deviation in a critical parameter can be of the order of $4 \%$, when measured only at the ND. See figure 4. However, and this is our key result, the double ratios are below $1.2 \%$. 

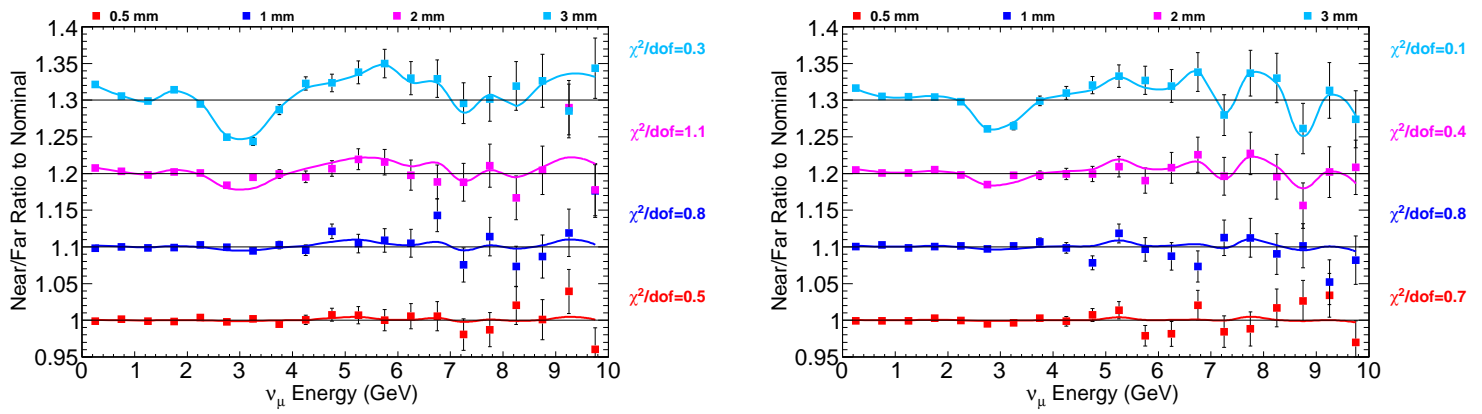

FIGURE 2. ND/FD double ratios for several values of the parameter that sets the target position along the horizontal axis, perpendicular to the beam direction (left plot). Also shown is the effect of a tilt of the target in the X-Z plane (right plot). These are "stacked" plots, where this double ratio is shifted upwards by 0.1 , for each separate value of the parameter, for graphical clarity.
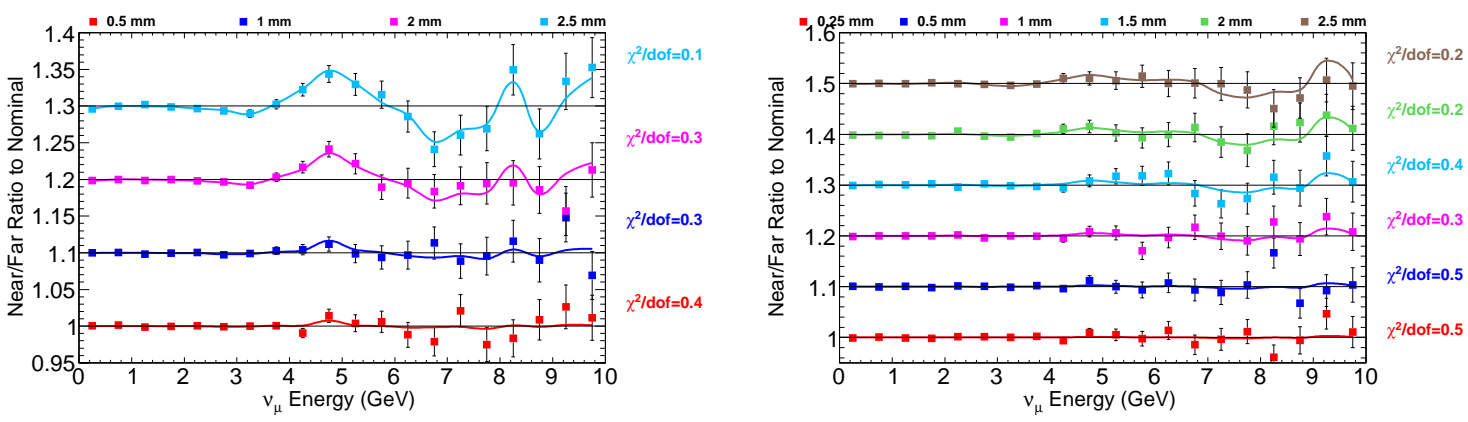

FIGURE 3. ND/FD double ratios for several values of the parameters that describe the misalignment of Horn 1 with respect to the nominal beam axis. Left: horizontal displacement Right: Tilt.

These figures have been compared to the expected statistical uncertainties in the far Detector. This is shown on figure 5 .
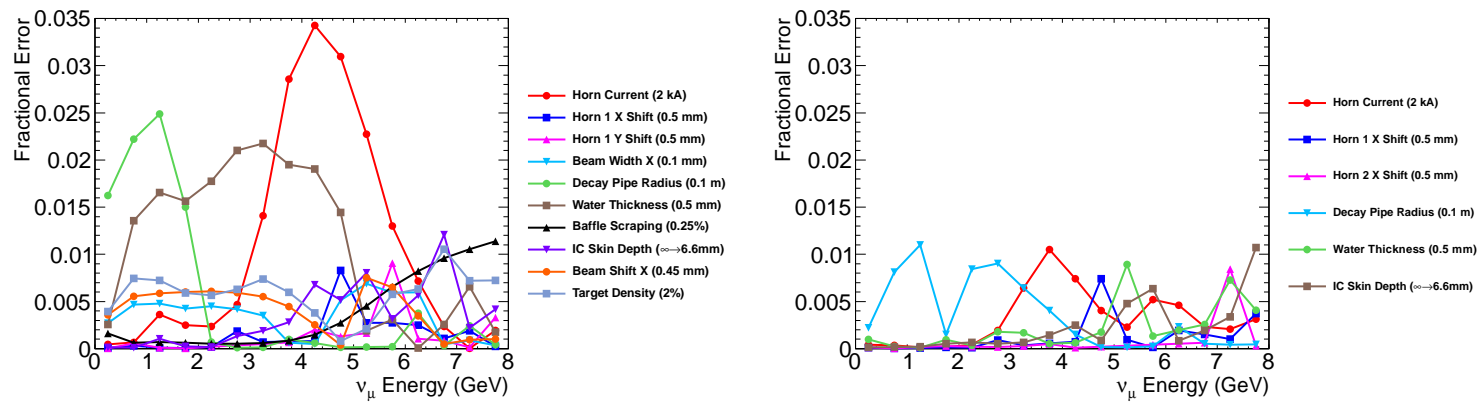

FIGURE 4. Left: The fractional uncertainty in the flux at the near detector, for the most critical alignment paramters and tolerances of the beamline components. Right: The ND/FD double ratios for the same set of parameters. 


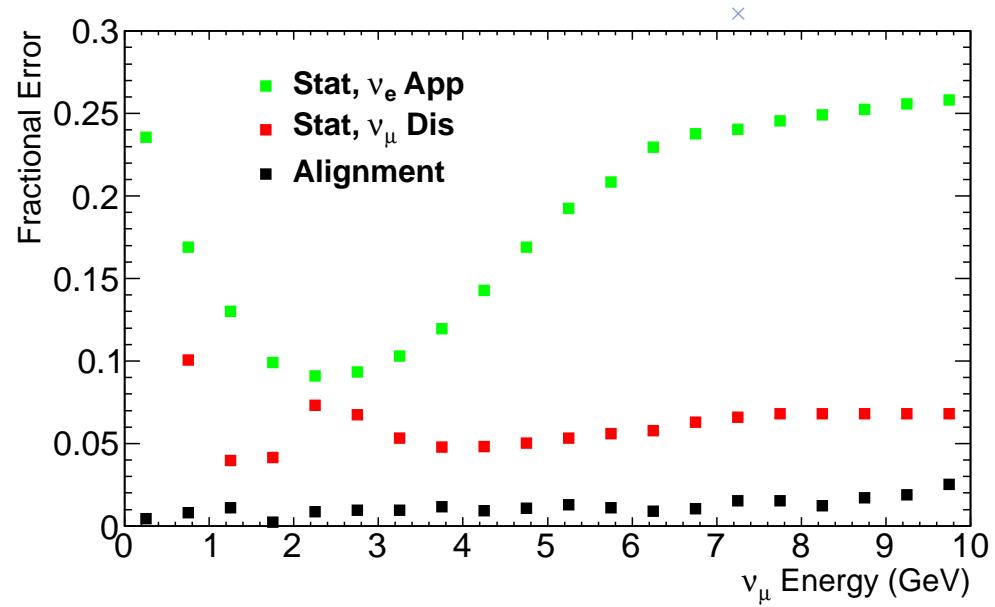

FIGURE 5. The fractional uncertainties, systematics (misalignments, horn current, geometry, etc..), and statistical, corresponding to a hypothetical run of $122.5 \mathrm{MW}$ kt years at the far Detector.

\section{CONCLUSIONS}

The G4LBNE package has been upgraded to support the determination of the systematics uncertainties due to misalignments of numerous beam line elements, as well as other uncertainties, such as the horn current and the skin depth of the aluminum alloy used for the inner conductors of the horns. The Geant 4 geometry has been completely re-implemented and verified with detailed and explicit dumps of straight trajectories, and visualization.

This work has been done for the so-called 700kW option of the existing NuMI beam line, for an early proposed LBNE baseline length and detectors, near and far. However, we do anticipate that the method will be directly applicable to the final LBNF beam line design, and, for the most part, the tolerance figures listed here will remain valid.

This calculation shows that, with a near detector, the systematic uncertainties on far/near detector flux ratios will be of the order of $1 \%$, over the relevant neutrino energy spectrum, for the measurement of the muon disappearance signal.

\section{ACKNOWLEDGMENTS}

This work could not have been done without the help and guidance for many members of the LBNE Beam Simulation group. In particular, we readily acknowledge the important contributions of Vaia Papadimitriou, Thomas junk, John LoSecco, Alysia Marino, Brett Viren, Jaehoon Yu and Bob Zwaska.

\section{REFERENCES}

1. C. Adams et al, The LBNEScience Document, BNL-101354-2014-JA, FERMILAB-PUB-14-022, LA-UR-14-20881 available at https://sharepoint.fnal.gov/project/lbne /LBNE\%20at\%20Work/LBNE\%20Science\%20Program/SitePages/Home.aspx

2. See reference above, p. 64.

3. S. Agostinelli et Al Nuclear Instruments and Methods in Physics Research A 506 (2003) 250-303 See also IEEE Transactions on Nuclear Science 53 No. 1 (2006) 270-278.

4. K. Anderson et al FERMILAB-DESIGN-1998-01, 1998.

5. N. V. Mokhov, P. Aarnio, Yu.I. Eidelman, K.K. Gudima, A.Yu. K onobeev, V.S. Pronskikh, I.L. Rakhno, S.I. Striganov, I.S. Tropin, 201cMARS15 code developments dri ven by the intensity frontier needs 201d, Fermilab- Conf-12-635-APC, Presented paper at the 12th Internationa 1 Conference on Radiation Shielding, September 2-7, 2012, Nara, Japan [1]

6. "NuMI Technical Design Handbook," http://www-numi.fnal.gov/numwork/tdh/tdh_index.html, see Chapter 4.6 